\title{
Individually and Combined Water-Based Exercise With Ginger Supplement, on Systemic Inflammation and Metabolic Syndrome Indices, Among the Obese Women With Breast Neoplasms
}

\author{
Niloofar Karimi, ${ }^{1, *}$ Valiollah Dabidi Roshan, and Zohreh Fathi Bayatiyani ${ }^{1}$ \\ ${ }^{1}$ Department of Sport Physiology, Faculty of Physical Education and Sport Sciences, University of Mazandaran, Babolsar, IR Iran \\ *Corresponding author: Niloofar Karimi, University of Mazandaran(UMZ), Pasdaran Street, 47415, P. O. Box: 416, Babolsar, IR Iran. Tel:+98-1125232091-95, E-mail: niloofarkarimi85@yahoo.com
}

Received 2015 August 23; Revised 2015 September 16; Accepted 2015 November 15.

\begin{abstract}
Background: Breast neoplasms has known as the most common cancer among the women worldwide, and relationship between obesity, metabolic syndrome, inflammation and cancer has been recognized since many years ago.

Objectives: The aim of this study was to determine the individual and concomitant effect of 6-weeks water-based exercise and oral ginger supplement on markers that have related to metabolic syndrome and systemic inflammation in obese women with breast neoplasms. Patients and Methods: Forty women whose have diagnosed with breast neoplasms have volunteered to participate in the study. Subjects have randomly assigned into four groups; placebo, exercise training, ginger supplement and exercise training+ ginger supplement groups. Subjects in the ginger supplement group and the exercise training+ ginger supplement group have orally received 4 capsules, 7 days a week and for 6 weeks. The water-based exercise training program have collected at a progressive intensity and time, have ranged from $50 \%$ to $75 \%$ of heart rate reserve, in a pool, 4 times a week for 6 weeks. Fasting blood sampling has collected at the pretest and post-test. Results: The ginger supplementation and the water-base exercise have resulted in a reduction of hs-CRP, IL-10, insulin, glucose, insulin resistance, LDL-C, TG; but an increase in HDL-C and HDL-C/LDL-C. The water-base exercise and ginger supplement group have significantly shown larger positive effect in all outcomes, in comparison with the water-base exercise or ginger supplement alone groups.

Conclusions: Findings have suggested that obese breast neoplasms survivors have commonly shown metabolic syndrome and elevated inflammation, which placed them at an increased risk for cardiovascular diseases. Moreover, data has indicated a protective effect of the nondrug strategies, such as water-base exercise and ginger supplementation have played an important role in pathogenesis of inflammatory and metabolic responses, among diagnosed breast neoplasms.
\end{abstract}

Keywords: Breast Neoplasms, Ginger Supplement, Water-Base Exercise, Inflammation, Metabolic Syndrome

\section{Background}

Recent epidemiological studies have revealed that overweight, particularly when characterized by central adiposity, has associated with elevated levels of proinflammatory factors, as well as the subsequent development of metabolic syndrome (MS), a clustering of metabolic disturbances that increased diseases $(1,2)$. Recently studies have suggested that breast neoplasms has associated with metabolic syndrome (3) and inflammation $(1,4)$, both associated to sedentary life and energy-dense diet (5). A healthy lifestyle index score (HLIS) has generated to investigate the joint effect of modifiable lifestyle factors among the postmenopausal breast cancer risk (5).

In addition to the direct benefits of physical activity for breast neoplasms, physical activity could also reduce adiposity $(6,7)$, which has associated with increased risk of lifetime breast neoplasms in postmenopausal women (8, 9). Also, exercise interventions have implemented concurrent with breast neoplasms treatment could cause increase in cardiovascular fitness, muscular strength, and body composition and decrease in cancer-related fatigue $(10,11)$. Various researchers have reported that water-based exercise is an ideal form of exercise for obese patients and might provide an attractive alternative to land-based exercise, to achieve improved health and fitness among these populations $(12,13)$.

The currently available therapeutic options such as dietary modification or a combination of synthetic antidiabetic, hypolipidaemic drugs have shown their own limitations and undesirable side-effects (2). Hence, there was an increased demand to search and evaluate traditional approaches for treatment of metabolic disorders, particularly the use of herbal medicines. Zingiber officinale, commonly known as ginger, has used in Ayurvedic and Chinese medicine for the treatment of metabolic syndrome, asthma, diabetes, nausea, pain and inflammation $(2,14$, 15). The role of oral ginger consumption as an anti-obesity, anti-metabolic syndrome, anti-cancer and anti-inflammatory factor in humans has not sufficiently studied.

Copyright (C) 2015, Iranian Journal of Cancer Prevention. This is an open-access article distributed under the terms of the Creative Commons Attribution-NonCommercial 4.0 International License (http://creativecommons.org/licenses/by-nc/4.0/) which permits copy and redistribute the material just in noncommercial usages, provided the original work is properly cited. 


\section{Objectives}

Thus, regarding to situation of these patients, whose need an exhilarating milieu, and the effect of physical activity in attractive environments like buoyant milieu, the purpose of this study was to determine the individual and concomitant effect of six-weeks water-based intermittent exercise in the pool, and oral ginger supplement on markers related to MS and pro-inflammatory markers in obese women suffering from breast neoplasms.

\section{Patients and Methods}

Forty obese women have diagnosed with breast neoplasms, volunteered to participate in the study. Prior to participation, all subjects have read and signed an informed consent document consistent with the guideline established by the ethics committee of the university of Mazandaran. All participants have completed radiation and/or chemotherapy treatments. Participants received comprehensive screening followed by an initial medical examination before they have included in the study. The characteristics of subjects have shown in Table 1.

Inclusion and exclusion criteria have included a primary diagnosis of breast neoplasms during stage I-II, no distant metastases, women have excluded if they had evidence of recurrent disease, have previously engaged in any formal exercise programs for at least six months prior to participation in this study. Also, according to medical examinations, the subject hasn't shown any cardiovascular and diabetic diseases. In addition, they have not smoked at least six months before beginning the study.

Subjects have randomly divided into four groups consisted to; 1) placebo, 2) exercise training, 3) ginger supplement and 4) exercise training+ ginger supplement groups (10 cases in each group). The subjects' anthropometric variables have included height; age, and body mass, percent of body fat, lean body mass, and body mass index (BMI) have assessed.

Subjects in the placebo and ginger supplement groups have not participated in any exercise program. However, each subject in the exercise training group and exercise training+ ginger supplement group have participated in four familiarization sessions. Each exercise session has generally included a 10 minutes warm up, 20 - 60 minutes of aerobic exercise in water and concluded with a 10-minute cool down.

Deep-water exercise protocol has defined as those in which participants had no foot contact with the bottom of the pool (12). This exercise program has implemented for 6 weeks and 4 sessions at a week. Exercise intensity has based on the treadmill assessment results, and ranged from $50 \%$ to $75 \%$ of heart rate reserve (HRR). Percentage of HRR has used to determine exercise heart rate intensity by using the formula (exercise target heart rate $=[\max$ HR - rest HR] $\times \%$ exercise intensity + rest HR (11).

After every set, the exercise intensity has controlled with pulse rate. The rests time between the sets was 5 to 6 minutes. Generally, the participants have exercised two sets daily during the first week, but for the next weeks, they have exercised three sets daily.

Subjects in the ginger supplement group and the exercise training ginger supplement group have orally received 4 capsules (Each capsule contained $750 \mathrm{mg}$ ) of ginger rhizome powder, 7 days a week and for 6 weeks, consistent with previous studies (16) The subjects have consumed the powder in approximately $250 \mathrm{ml}$ of water. The supplement has ingested 4 times a day, with breakfast, lunch, dinner and afternoon. The placebo group has received capsules contained 1 gr starch, in the same manner and for the same duration of time as the experimental groups. Blood samples have collected from each subject at pretest and at the end of 6 weeks of water-based exercise with and without ginger supplement in an overnight 10 to12-h fasting state. The blood samples have allowed to clot at room temperature for $10 \mathrm{~min}$ and then centrifuged. The hs-CRP concentration has determined by Latex particle-enhanced, as described by Roshan et al. (17). Also, IL-10 has assessed by commercially available enzyme immunesorbent assay (ELISA) kit.

Furthermore, Fasting serum triglycerides (TG), total cholesterol (TC), high-density lipoprotein cholesterol (HDL-C) and low-density lipoprotein cholesterol (LDL-C) concentrations have measured by an enzymatic colorimetric method. Blood glucose levels have measured via a hexokinase enzymatic reference method. Furthermore, Plasma insulin levels have measured using an immunoradiometric assay. Also, the homeostasis model assessment for insulin resistance (HOMA-IR) as determined by the following equation (3):

HOMA-IR $=$ Fasting insulin $\left(\frac{\mu \mathrm{U}}{\mathrm{mL}}\right) \times \frac{\text { fasting } \text { glucose }\left(\frac{\mathrm{mmol}}{\mathrm{L}}\right)}{22.5}$

Statistical analysis has performed SPSS version 16.0. Differences have considered significant at $\mathrm{P}<0.05$.

\begin{tabular}{|c|c|c|c|c|c|}
\hline Parameter and Groups & Placebo & Ginger & Exercise & Ginger + Exercise & P Value \\
\hline Age, $y$ & $50.4 \pm 3.4$ & $46.4 \pm 5.5$ & $47.3 \pm 8.1$ & $47.5 \pm 4.6$ & .434 \\
\hline Height, m & $1.56 \pm 4.9$ & $1.57 \pm 4.6$ & $1.57 \pm 6.7$ & $1.56 \pm 4$ & .949 \\
\hline Weight, kg & $74.27 \pm 10.9$ & $77.61 \pm 7.5$ & $71.36 \pm 4.9$ & $74.74 \pm 9$ & .435 \\
\hline Fat mass, $\%$ & $42.48 \pm 3.9$ & $39.2 \pm 3.9$ & $40.52 \pm 5.5$ & $40.1 \pm 3.3$ & .381 \\
\hline BMI, $\mathrm{kg} / \mathrm{m}^{2}$ & $32.77 \pm 2.9$ & $32.18 \pm 2.9$ & $29.78 \pm 3$ & $31.26 \pm 5.1$ & .289 \\
\hline
\end{tabular}




\section{Results}

Mean values of the subjects characteristics (age, height, body mass, BMI and fat mass) for the four groups have shown in Table 1. At first, no differences have existed in the aforesaid characteristic between groups. Changes in the inflammatory markers (hs-CRP and IL-10) and biomarkers of the MS (insulin, glucose, insulin resistance, blood lipids such as; LDL-C, HDL-C, TC, TG) in women with breast neoplasms in each group have shown in Table 2. Six weeks of the ginger supplementation have resulted in a reduction of hs-CRP $(\mathrm{P}=0.002)$, IL-10 $(\mathrm{P}=0.001)$, insulin $(\mathrm{P}=0.516)$, glucose $(\mathrm{P}=0.003)$, insulin resistance $(\mathrm{P}=$ $0.005)$, LDL-C $(\mathrm{P}=0.432), \mathrm{TG}(\mathrm{P}=0.851)$ and an increase in TC $(P=0.514)$, and another increase in HDL-C $(P=0.716)$ and HDL-C/LDL-C $(P=0.264)$, in comparison with the period of time before 6-wk ginger supplementation (Table 2). In contrast, 6-wk of the water-base exercise has caused a reduction of hs-CRP $(\mathrm{P}=0.059)$, IL-10 $(\mathrm{P}=0.054)$, insulin $(P=0.035)$, glucose $(P=0.051)$, insulin resistance $(P$ $=0.003)$, LDL-C $(P=0.094), \mathrm{TC}(\mathrm{P}=0.300), \mathrm{TG}(\mathrm{P}=0.031)$ and an increase in HDL-C $(\mathrm{P}=0.033)$, and HDL-C/LDL-C $(\mathrm{P}=$ 0.012 ), in comparison with the period of time before 6 -wk water-base exercise. However, the water-base exercise + ginger treatment was more effective than the water-base exercise and the ginger supplement, alone treatment, such that, it has led to a reduction of hs-CRP $(\mathrm{P}=0.001)$,
IL-10 $(\mathrm{P}<0.001)$, insulin $(\mathrm{P}=0.008)$, glucose $(\mathrm{P}=0.036)$, insulin resistance $(\mathrm{P}=0.002)$, $\mathrm{LDL}-\mathrm{C}(\mathrm{P}=0.003), \mathrm{TC}(\mathrm{P}=$ $0.654), \mathrm{TG}(\mathrm{P}=0.003)$ and an increase in $\operatorname{HDL}-\mathrm{C}(\mathrm{P}=0.010)$ and HDL-C/LDL-C $(\mathrm{P}<0.001)$, in comparison with 6-wk treatment (Tables 2 and 3).

Furthermore, the ginger supplement resulted in a significant decrease in hs-CRP $(\mathrm{P}<0.001)$ and IL-10 $(\mathrm{P}<0.001)$ Levels, in comparison with the placebo group. Also, significant decreases in hs-CRP and IL-10 Levels were present, not only in the water-base exercise group $(\mathrm{P}=0.010$ and $\mathrm{P}=0.001$, respectively), but decreases have also observed in the water-base exercise + ginger supplement group ( $\mathrm{P}$ $<0.001$ and $\mathrm{P}<0.001$, respectively), in comparison with sedentary control group (Table 2 ).

The water-base exercise has significantly decreased the insulin resistance $(P=0.039)$, insulin $(P=0.030)$, and significantly increased HDL-C/LDL-C ( $P=0.011)$, in comparison with placebo group. In addition, it has caused insignificant decrease in LDL-C $(P=0.089)$ and insignificantly increased the HDL-C $(\mathrm{P}=0.061)$, in comparison with placebo group. The water-base exercise and ginger supplement group has shown significantly larger positive effect in all of the outcomes, in comparison with the water-base exercise or ginger supplement alone groups (Table 3).

\begin{tabular}{lcccc}
\hline \multicolumn{2}{l}{ Table 2. Levels of Systemic Inflammation After Water-Based Exercise, With and Without Ginger Supplement ${ }^{\mathrm{a}}$} \\
\hline Markers and Groups & Placebo & Ginger & Exercise & Ginger + Exercise \\
\hline IL-10, pg/mL & $47.5 \pm 4.5$ & $36.7 \pm 3.48^{\mathrm{b}}$ & $40.6 \pm 4.1^{\mathrm{b}}$ & $33.6 \pm 3.9^{\mathrm{b}}$ \\
hs-CRP, $\mathbf{~ m g / L}$ & $3.88 \pm 0.8$ & $2.49 \pm 0.6^{\mathrm{b}}$ & $2.89 \pm 0.5^{\mathrm{b}, \mathrm{c}}$ & $2.02 \pm 0.9^{\mathrm{b}}$ \\
\hline
\end{tabular}

${ }^{\mathrm{a}}$ Data have presented as the mean \pm SD for 10 subjects.

bignificantly different from placebo group.

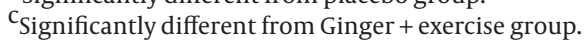

Table 3. Levels of the Metabolic Syndrome Markers After Water-Based Exercise, With and Without Ginger Supplement ${ }^{\mathrm{a}}$

\begin{tabular}{lcccc}
\hline Markers and Groups & Placebo & Ginger & Exercise & Ginger + Exercise \\
\hline Insulin, $\boldsymbol{\mu U} / \mathbf{m L}$ & $7.4 \pm 1.3$ & $7.1 \pm 1.2^{\mathrm{b}}$ & $5.9 \pm 1^{\mathrm{C}}$ & $5.7 \pm 1^{\mathrm{C}}$ \\
\hline Insulin resistance & $2.1 \pm 0.5$ & $2 \pm 0.4$ & $1.6 \pm 0.3^{\mathrm{C}}$ & $1.6 \pm 0.2^{\mathrm{b}}$ \\
\hline Glucose, $\mathbf{m m o l} / \mathbf{L}$ & $6.5 \pm 1$ & $6.5 \pm 0.4$ & $6.6 \pm 0.6$ & $6.5 \pm 0.7$ \\
TG, $\mathbf{m g} / \mathbf{d L}$ & $1.38 \pm 22.6$ & $1.45 \pm 35.8$ & $1.17 \pm 15$ & $1.16 \pm 13.9$ \\
TC, $\mathbf{m g} / \mathbf{d L}$ & $2.13 \pm 30.9$ & $1.92 \pm 19.3$ & $1.91 \pm 19.7$ & $1.85 \pm 25.5$ \\
\hline LDL-C, $\mathbf{m g} / \mathbf{d L}$ & $1.4 \pm 17.6$ & $1.3 \pm 17.7$ & $1.2 \pm 15.8$ & $1.2 \pm 16$ \\
\hline HDL-C, $\mathbf{~ m g / d L}$ & $44.5 \pm 5.1$ & $44.7 \pm 5.2^{\mathrm{b}}$ & $51.4 \pm 7.4$ & $53 \pm 5.6^{\mathrm{C}}$ \\
\hline HDL/LDL-C & $0.318 \pm 0.061$ & $0.33 \pm 0.08^{\mathrm{b}}$ & $0.41 \pm 0.06^{\mathrm{C}}$ & $0.43 \pm 0.06^{\mathrm{C}}$ \\
\hline
\end{tabular}

\footnotetext{
${ }^{\mathrm{a}}$ Data are presented as the mean \pm SD for 10 subjects.

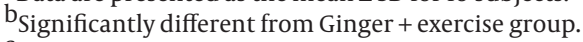

Cignificantly different from placebo group.
} 


\section{Discussion}

Weight gain has been a risk factor for postmenopausal breast neoplasms. In addition, a significant percentage of women whose have treated the breast neoplasms, have reported undesirable weight gain during and after treatment $(1,18)$. This study has designed to determine whether 6 weeks of individually, and combined water-based exercise with ginger supplement would influence the inflammatory markers and metabolic syndrome in obese women with breast neoplasms. The primary novel findings in present study have shown that consumption of ginger was beneficial in alleviating the systemic inflammation, insulin resistance and glucose, while it has not shown significant effect on TC, TG, insulin, LDL-C, HDL-C, and the HDL-C to LDL-C ratio.

On the other hand, 6 weeks of water-based exercise have caused significant reduce in insulin resistance, insulin, TG,HDL-C and HDL-C/LDL-C levels, and insignificant decrease in hs-CRP, IL-10, LDL-C, TC and glucose concentrations, in comparison with placebo group. However, the main findings of our study was that water-based exercise with ginger supplementation has significantly attenuated systemic inflammation and metabolic syndrome in obese women with breast neoplasms, in comparison with the other groups. These findings were consistent with data from Fernandez-Lao et al. (19) that has reported reduction in blood lipid, glucose and insulin response, and improvement in cardiovascular health following water versus land based exercise in breast cancer survivors. Overall, non-weight bearing exercise has recommended as a possible alternative to improve health and well-being for individuals who found weight bearing exercise difficult (12).

The regular physical activity might influence prognosis by similar mechanisms to those thought to prevent the incidence of breast neoplasms, including decreased lifetime estrogen exposure and improved immune function (20). Increased physical activity also has reduced insulin resistance and hyperinsulinemia. Furthermore, lower blood-estrogen levels, higher insulin growth factor-I concentration, and lower levels of fatty tissue have all been associated with participation in physical activity.

Specific obesity-associated factors, including blood lipids, insulin and inflammatory mediators, have seemed to influence breast neoplasms growth and prognosis independently of estrogens (21). Inflammatory cytokines have produced as acute phase proteins by the liver, and might provide much more accurate reflection of cytokine activity. IL- 6 has been a known inflammatory cytokine that has secreted by activated macrophage and involved in several functions of the immune response that has also exerted various metabolic and endocrine activities. Boonyaratanakornkit et al. (21) has reported the cessation of ovarian function after menopause results in withdrawal of ovarian sex steroid hormones, estrogen, and progesterone. Accumulating evidence has suggested that the withdrawal of estrogen and progesterone has caused homeostasis imbalances, including decreases in insulin sensitivity and changes in glucose and lipid metabolism, resulting in a total reduction in energy expenditure. Together with a decrease in physical activity and consumption of high fat diet, these factors have significantly contributed to obesity in postmenopausal women. Obesity has caused localized inflammation, an increase in local estrogen production, and changes in cellular metabolism. In addition, obese women have shown a higher risk of insulin insensitivity, and an increase in insulin, and other growth factor secretion $(1,3)$. The factors that have influenced inflammatory responses include age, gender, repeated bouts of eccentric exercise, antioxidant supplements, intracellular calcium homeostasis, and anti-inflammatory drugs (22).

Results from the few studies that have investigated the effects of water-based training on the markers related to metabolic syndrome have been inconsistent. Twelve weeks of shallow water circuit training has reported to significantly reduce TC and LDL-C levels with no significant changes in HDL-C or TG in older, overweight women. The lack of markedly change in some of the MS (LDL$\mathrm{C}$, TC and glucose) after chronic water-based training was a disagreement with the effects of inflammatory markers. The discrepant results might be due to the duration of the training programs enlisted, the intensity of the training program, or the baseline lipid status of the participants.

The present study has revealed that ginger supplementation has caused improvement in inflammation and metabolic syndrome, in comparison with the supplementation period, as well as the placebo group. In laboratory experiments, ethanolic extract of $Z$. officinale has shown to reduce plasma lipids in cholesterol-fed hyperlipidaemic rabbits and in streptozotoc in induced diabetic rats and has also found to inhibit LDL oxidation in atherosclerotic mice. Besides, the aqueous extract of $Z$. officinale has also shown to reduce serum cholesterol and triglycerides in normal rats (14).

Ginger has shown to inhibit the release of proinflammatory cytokines in vitro. Moreover, ginger has suppressed inflammation by inhibiting the nuclear factor- $\kappa \mathrm{B}$ activation, which led to inhibition of gene expression of proinflammatory cytokines, chemokines and cyclooxygenase (COX)-2 as well as osteoclastogenesis (23). In addition, ginger extract has found to inhibit beta-amyloid peptide-induced cytokine and chemokine expression in cell line of human monocytes (23). The question of whether ginger had large effects on pain in women with breast neoplasms will be best addressed by 
future studies, which used the same scale to assess pain perceptions.

In summary, our data has indicated a protective effect of the nondrug strategies such as water-base exercise and ginger supplementation has played an important role in the pathogenesis of inflammatory and metabolic responses in obese women, who have diagnosed with breast neoplasms. However, the combination of water-base exercise and ginger supplement has shown better effect on the systemic inflammatory and metabolic syndrome, rather than using each of them separately.

\section{Acknowledgments}

The authors of this paper have thanked Dr Mehran hoseynzadeh, for cooperation and scientific advice. In addition, would appreciate from the subjects and cooperation in research.

\section{Footnotes}

Authors' Contribution:All costs of research have provided by the authors. All authors have involved to gather the information and to write the paper.

Conflict of Interests:None declared.

Financial Disclosure:None declared.

\section{References}

1. Berrino F, Villarini A, Traina A, Bonanni B, Panico S, Mano MP, et al. Metabolic syndrome and breast cancer prognosis. Breast Cancer Res Treat. 2014;147(1):159-65. doi:10.1007/s10549014-3076-6. [PubMed: 25104441]

2. Nammi S, Sreemantula S, Roufogalis BD. Protective effects of ethanolic extract of Zingiber officinale rhizome on the development of metabolic syndrome in high-fat diet-fed rats. Basic Clin Pharmacol Toxicol. 2009;104(5):366-73. doi: 10.1111/j.1742-7843.2008.00362.x. [PubMed: 19413656]

3. Agnoli C, Grioni S, Sieri S, Sacerdote C, Ricceri F, Tumino R, et al. Metabolic syndrome and breast cancer risk: a casecohort study nested in a multicentre italian cohort. PLoS One. 2015;10(6):e0128891. doi: 10.1371/journal.pone.0128891. [PubMed: 26030767]

4. Alfano CM, Imayama I, Neuhouser ML, Kiecolt-Glaser JK, Smith AW, Meeske K, et al. Fatigue, inflammation, and omega-3 and omega- 6 fatty acid intake among breast cancer survivors. J Clin Oncol. 2012;30(12):1280-7. doi: 10.1200/ JCO.2011.36.4109. [PubMed: 22412148]

5. McKenzie F, Ferrari P, Freisling H, Chajes V, Rinaldi S, de Batlle J, et al. Healthy lifestyle and risk of breast cancer among postmenopausal women in the European Prospective Investigation into Cancer and Nutrition cohort study. Int $J$ Cancer. 2015;136(11):2640-8. doi: 10.1002/ijc.29315. [PubMed: 25379993]

6. Hartman SI, Dunsiger SI, Jacobsen PB. The relationship of psychosocial factors to mammograms, physical activity, and fruit and vegetable consumption among sisters of breast cancer patients. Int J Womens Health. 2011;3:257-63. doi: 10.2147/IJWH.S23246. [PubMed: 21892336]

7. Jakicic JM, Tate DF, Lang W, Davis KK, Polzien K, Neiberg RH, et al. Objective physical activity and weight loss in adults: the step-up randomized clinical trial. Obesity (Silver Spring). 2014;22(11):2284-92. doi: 10.1002/oby.20830. [PubMed: 25376395]

8. Patterson RE, Cadmus LA, Emond JA, Pierce JP. Physical activity, diet, adiposity and female breast cancer prognosis: a review of the epidemiologic literature. Maturitas. 2010;66(1):515. doi:10.1016/j.maturitas.2010.01.004. [PubMed: 20097494]

9. Renehan AG, Tyson M, Egger M, Heller RF, Zwahlen M. Bodymass index and incidence of cancer: A systematic review and meta-analysis of prospective observational studies. Lancet. 2008;371(9612):569-78. doi: 10.1016/S0140-6736(08)60269-X. [PubMed: 18280327]

10. Sprod LK, Hsieh CC, Hayward R, Schneider CM. Three versus six months of exercise training in breast cancer survivors. Breast Cancer Res Treat. 2010;121(2):413-9. doi: 10.1007/s10549010-0913-0. [PubMed: 20443054]

11. Schneider CM, Hsieh CC, Sprod LK, Carter SD, Hayward R. Effects of supervised exercise training on cardiopulmonary function and fatigue in breast cancer survivors during and after treatment. Cancer. 2007;110(4):918-25. doi: 10.1002/ cncr.22862. [PubMed: 17582616]

12. Meredith-Jones $\mathrm{K}$, Waters $\mathrm{D}$, Legge $\mathrm{M}$, Jones L. Upright water-based exercise to improve cardiovascular and metabolic health: A qualitative review. Complement Ther Med. 2011;19(2):93-103. doi: 10.1016/j.ctim.2011.02.002. [PubMed: 21549260]

13. Bento PC, Lopes Mde F, Cebolla EC, Wolf R, Rodacki AL. Effects of Water-Based Training on Static and Dynamic Balance of Older Women. Rejuvenation Res. 2015;18(4):326-31. doi: 10.1089/rej.2014.1650. [PubMed: 25708712]

14. Young HY, Luo YL, Cheng HY, Hsieh WC, Liao JC, Peng WH. Analgesic and anti-inflammatory activities of [6]-gingerol. J Ethnopharmacol. 2005;96(1-2):207-10. doi: 10.1016/j. jep.2004.09.009. [PubMed: 15588672]

15. Zaman SU, Mirje MM. Evalutation of the anti-inflamatory effect of zingiber officinale (Ginger) root in rats. Citeseer. 2014;3(1):2250-62.

16. Black CD, Herring MP, Hurley DJ, O'Connor PJ. Ginger (Zingiber officinale) reduces muscle pain caused by eccentric exercise. J Pain. 2010;11(9):894-903. doi: 10.1016/j. jpain.2009.12.013. [PubMed: 20418184]

17. Roshan VD, Assali M, Moghaddam AH, Hosseinzadeh M, Myers J. Exercise training and antioxidants: effects on rat heart tissue exposed to lead acetate. Int J Toxicol. 2011;30(2):190-6. doi: 10.1177/1091581810392809. [PubMed: 21378372]

18. Jones LM, Legge M, Meredith-Jones K. Circuit based deep water running improves cardiovascular fitness, strength and abdominal obesity in older, overweight women aquatic exercise intervention in older adults. Medicina Sportiva. 2009;13(1):5-12. doi: 10.2478/v10036-009-0002-9.

19. Fernandez-Lao C, Cantarero-Villanueva I, Ariza-Garcia A, Courtney C, Fernandez-de-las-Penas C, Arroyo-Morales M. Water versus land-based multimodal exercise program effects on body composition in breast cancer survivors: a controlled clinical trial. Support Care Cancer. 2013;21(2):521-30. doi: 10.1007/s00520-012-1549-x. [PubMed: 22864470]

20. Brenner DR, Neilson HK, Courneya KS, Friedenreich CM. Physical Activity After Breast Cancer: Effect on Survival and Patient-Reported Outcomes. Curr Breast Cancer Reports. 2014;6(3):193-204. doi: 10.1007/s12609-014-0147-y.

21. Boonyaratanakornkit V, Pateetin P. The role of ovarian sex steroids in metabolic homeostasis, obesity, and postmenopausal breast cancer: molecular mechanisms and therapeutic implications. Biomed Res Int. 2015;2015:140196. doi: 10.1155/2015/140196. [PubMed: 25866757]

22. Bower JE, Ganz PA, Tao ML, Hu W, Belin TR, Sepah S, et al. Inflammatory biomarkers and fatigue during radiation 


\section{Karimi N et al.}

therapy for breast and prostate cancer. Clin Cancer Res. 2009;15(17):5534-40. doi: 10.1158/1078-0432.CCR-08-2584. [PubMed: 19706826]
23. Peake J, Nosaka K, Suzuki K. Characterization of inflammatory responses to eccentric exercise in humans. Exerc Immunol Rev. 2005;11:64-85. [PubMed: 16385845] 